\title{
Diagnosi e valutazione della personalità, alleanza terapeutica e scambio clinico nella ricerca in psicoterapia
}

\author{
Vittorio Lingiardi, ${ }^{1}$ Francesco Gazzillo, ${ }^{1}$ Antonello Colli, ${ }^{2}$ \\ Francesco De Bei, ${ }^{1}$ Annalisa Tanzilli, ${ }^{1}$ Mariagrazia Di Giuseppe, ${ }^{1}$ \\ Nicola Nardelli, ${ }^{1}$ Chiara Caristo,${ }^{1}$ Valeria Condino, ${ }^{1}$ Daniela Gentile,${ }^{1}$ \\ Nino Dazzi ${ }^{1}$
}

\section{Sommario}

Questo contributo si propone di fornire una breve rassegna delle principali linee di ricerca seguite negli ultimi anni dal gruppo coordinato da Vittorio Lingiardi. Tra queste, ci soffermeremo in particolare su: a) valutazione e diagnosi della personalità con SWAP-200 e PDM (Psychodynamic Diagnostic Manual); b) sviluppo e validazione di strumenti clinician-report per operazionalizare l'uso del PDM; c) valutazione dei meccanismi di difesa e degli stili difensivi mediante DMRS e sua versione Q sort; d) studio del processo e della relazione terapeutica (alleanza terapeutica, rotture e riparazioni dell'alleanza, transfert, controtransfert); in particolare, in quest'area di ricerca, ci siamo impegnati nello sviluppo e validazione di nuovi strumenti per la valutazione dei processi di rottura e riparazione dell'alleanza (Collaborative Interaction Scale) e della qualità dell'attaccamento tra paziente e terapeuta (Patient-Therapist Attachment $Q$ Sort); d) sviluppo della ricerca clinica e applicativa sui temi dell'identità di genere, dell'orientamento sessuale e dell'omofobia sociale e interiorizzata.

\section{Parole chiave}

Diagnosi e valutazione della personalità, meccanisimi di difesa, processo, alleanza terapeutica, orientamento sessuale e omofobia.

${ }_{1}$ Dipartimento di Psicologia Dinamica e Clinica, Facoltà di Medicina e Psicologia, "Sapienza", Università di Roma, ${ }^{2}$ Dipartimento di Scienze dell’Uomo, Università degli Studi di Urbino "Carlo Bo"

Corrispondenza: Vittorio Lingiardi

E-mail: vittorio.lingiardi@uniroma1.it

Tel.: 0649917559 


\section{Introduzione}

Il nostro approccio alla "ricerca in psicoterapia" può essere riassunto in pochi concetti essenziali: che la ricerca sia utile alla clinica, che la clinica sia capace di riflettere su se stessa a partire dai risultati della ricerca, che si rivolga la stessa attenzione alla complessità dei concetti e dei fenomeni e all'affidabilità empirica della loro operazionalizzazione, che si nutra fiducia in numeri e tabelle, ma senza idealizzarli, che si dia una considerazione privilegiata alle ricerche basate sui trascritti delle sedute e che si usino poche gergalità.

Le nostre linee di ricerca possono essere così raggruppate: 1) valutazione e diagnosi della personalità; 2) relazione terapeutica e sue componenti (alleanza terapeutica, transfert, controtransfert); 3) attaccamento e psicoterapia; 4) valutazione dei meccanismi di difesa; 5) orientamento sessuale, omofobia, identità di genere e psicoterapia.

In questa breve rassegna sull'attività di ricerca del nostro gruppo faremo riferimento prevalentemente alla produzione scientifica degli ultimi anni. Abbiamo deciso di riportare in forma narrativa i risultati delle nostre ricerche, rimandando ai singoli lavori il lettore interessato a informazioni più specifiche sulle tecniche di analisi utilizzate e i dati numerici (informazioni aggiornate e complete sulle attività di ricerca del nostro gruppo sono consultabili sul sito: http://www.pcrlab.org/).

\section{Valutazione e diagnosi della personalità}

Dopo una rassegna critica dei principali modelli della personalità e dei suoi disturbi (Lingiardi, 2004), in particolare da una prospettiva psicodinamica e bio-psico-sociale, abbiamo cercato di individuare uno strumento di valutazione della personalità che fosse al tempo stesso empiricamente affidabile e clinicamente utile, che superasse i principali limiti dell'Asse II del DSM-IV-TR (APA, 2000) e che potesse essere utilizzato anche nelle ricerche su processo e outcome delle psicoterapie. 
Abbiamo riscontrato questi requisiti nella Shedler-Westen Assessment Procedure-200, un Q-sort clinical-report composto da 200 item descrittivi delle principali caratteristiche della personalità normale e patologica (Gazzillo, 2006, 2009; Lingiardi \& Gazzillo, 2002, 2010; Westen \& Shedler, 1999a, 1999b; Westen, Shedler, \& Lingiardi, 2003). La SWAP-200 permette di elaborare una diagnosi categoriale e dimensionale degli stili/disturbi di personalità che segue la nosografia dell'Asse II del DSM-IV (fattori PD) e una tassonomia empiricamente derivata degli stili di personalità (fattori Q), una valutazione delle risorse e una formulazione del caso sistematica e patient-tailored. A differenza degli strumenti self-report, la SWAP-200 valorizza le capacità di osservazione e inferenza dei clinici, disciplinandole per mezzo di metologie statistiche adeguate e senza interferire nella relazione terapeuta-paziente.

Abbiamo tradotto in italiano la SWAP-200, versioni adulti e adolescenti (per quest'ultima vedi: Westen, Shedler, Durrett, Glass, \& Martens, 2003), e costruito un programma computerizzato per lo scoring (Westen et al., 2003).

Dal punto di vista applicativo, ci siamo concentrati sull'impiego della SWAP-200 nella valutazione di processo e outcome delle psicoterapie per mezzo dell'analisi dei trascritti di sedute di casi singoli: in un primo lavoro pubblicato sul Journal of Personality Assessment (Lingiardi, Shedler, \& Gazzillo, 2006) abbiamo illustrato come la SWAP-200 permetta di valutare in modo dettagliato, qualitativo e quantitativo, i cambiamenti dell'assetto della personalità nel corso di una psicoterapia analitica. In un lavoro più recente, apparso su Psychoanalytic Psychology (Lingiardi, Gazzillo, \& Waldron, 2010), abbiamo invece analizzato processo e outcome di una psicoterapia psicoanalitica servendoci di SWAP-200, Defense Mechanism Rating Scale (DMRS; Perry, 1990) e Analytic Process Scales (APS; Waldron, Scharf, Hurst, Firestein, \& Burton, 2004) per la valutazione delle comunicazioni di 
paziente e terapeuta. I risultati di questa ricerca sembrano confermare alcuni dati presenti in letteratura (Waldron, Scharf, Hurst et al., 2004; Waldron, Scharf, Crouse, Firestein, Burton, \& Hurst, 2004; Waldron \& Helm, 2005): una terapia di successo aumenta le capacità di buon funzionamento della personalità del paziente, riduce i tratti di personalità patologici e matura complessivamente lo stile difensivo. Questo studio sembra anche supportare l'ipotesi secondo cui le attività analitiche nucleari (chiarificazioni e interpretazioni di difese, conflitti e transfert) e la qualità delle comunicazioni del terapeuta (intesa come adeguatezza del tipo e del contenuto dell'intervento, a cui aggiungere tatto, timing e linguaggio con cui viene comunicato) sono i fattori che maggiormente favoriscono il cambiamento della personalità nelle terapie analitiche.

Attualmente, siamo impegnati nella stesura di un lavoro di valutazione del processo di una psicoterapia dinamica ai cui trascritti sono state applicate SWAP-200, Attività Referenziale (RA; Bucci \& Kabasakalian-Mckay, 1992) e DMRS (per una versione preliminare, vedi Gazzillo, Mariani, Lingiardi, \& Bucci, 2009).

In una ricerca empirica condotta su 102 adolescenti con disturbi del comportamento alimentare (DCA), abbiamo utilizzato la SWAP-200 per identificare $i$ sottotipi di personalità empiricamente rilevabili nei pazienti con queste diagnosi. In linea con le ricerche di ThompsonBrenner e Westen (Thompson-Brenner, Eddy, Franko, Dorer, Vashchenko, Kass, \& Herzog, 2008; Thompson-Brenner \& Westen, 2005), abbiamo identificato tre sottotipi di personalità: uno stile di alto funzionamento/perfezionistico, uno emotivamente disregolato e uno iper-coartato. Abbiamo quindi indagato le caratteristiche relative all'identità e allo stile di regolazione degli affetti di ciascuno di questi sottotipi e rilevato come il livello di gravità della presentazione clinica dei pazienti con DCA sia direttamente correlato all'intensità dello stile disregolato e inversamente correlato a quello di alto funzionamento 
(Gazzillo, Lingiardi, Peloso, Giordani, Vesco, Filippucci, \& Zanna, submitted). Attualmente, in collaborazione con il SERT della ASL di Bergamo, stiamo conducendo uno studio finalizzato alla sottotipizzazione della personalità di pazienti con addiction.

In ultimo, con i colleghi del Terzo Centro di Psicoterapia Cognitiva di Roma abbiamo avviato un progetto di ricerca teso a indagare la relazione tra patologia di personalità (valutata con la SWAP-200; Westen et al., 2003), pattern transferali (identificati con il Psychotherapy Relationship Questionnaire (PRQ); Westen, 2000; Bradley, Heim, \& Westen, 2005) e pattern controtransferali (identificati con il Countertransference Questionnaire (CTQ); Zittel, Conklin, \& Westen, 2003; Betan, Heim, Conklin, \& Westen, 2005).

In linea con le ipotesi formulate, è emerso che il transfert evitante/contro-dipendente e il controtransfert distaccato sembrano buoni predittori dei disturbi di personalità del cluster A; il transfert arrabbiato/rivendicativo/recriminativo e il controtransfert sopraffatto/disorganizzato sembrano buoni predittori dei disturbi del cluster B; il transfert ansioso/preoccupato e il controtransfert genitoriale sembrano buoni predittori dei disturbi del cluster C.

Questi risultati suggeriscono che le modalità con cui pazienti e terapeuti interagiscono presentano caratteristiche riconducibili alla personalità del paziente e tendono a ripetersi secondo pattern coerenti e prevedibili (Tanzilli, Carcione, Dimaggio, Lingiardi, \& Semerari, 2010).

Nel 2008, con Franco Del Corno, abbiamo curato la versione italiana del Manuale Diagnostico Psicodinamico (PDM Task Force, 2006), il primo manuale multiassiale, catagoriale, dimensionale e prototipico pensato per guidare il clinico in una valutazione dinamica completa della personalità, del funzionamento mentale e della sintomatologia (incluso il senso soggettivo dei sintomi) di pazienti adulti, adolescenti e bambini. Per validare empiricamente la tassonomia dei disturbi di 
personalità del PDM e permettere una loro valutazione rapida e affidabile abbiamo costruito i Prototipi Diagnostici Psicodinamici (PDP; Gazzillo, Lingiardi, \& Cordero di Montezemolo, 2008), una rating scale clinician-report dei diversi disturbi di personalità del PDM. La validazione dei PDP, condotta su 173 soggetti adulti in cura presso i servizi pubblici di salute mentale, ha dimostrato buoni livelli di validità di facciata, attendibilità, validità concordante, discriminante e di criterio (Gazzillo, Lingiardi, \& Del Corno, in preparazione). Siamo impegnati nell'elaborazione di un modello di formulazione dei casi clinici basato sugli item dei PDP e nella costruzione di un programma computerizzato per la valutazione della personalità con questo strumento, che nella sua ultima versione (Gazzillo \& Lingiardi, 2010) indaga anche le preoccupazioni principali e le credenze patogene dei pazienti, prima valutate per mezzo di due questionari ad hoc.

L'attenzione alla diagnosi e la promozione di una cultura diagnostica propedeutica alle opzioni di trattamento, ci ha portato a curare, su commissione dell'Ordine degli Psicologi del Lazio, il primo testo italiano di rassegna critica dei principali modelli e strumenti diagnostici di ambito psicologico-clinico: La diagnosi in psicologia clinica (Dazzi, Lingiardi, \& Gazzillo, 2009; vedi anche Barron \& Lingiardi, 2005; Lingiardi, 2009; Lingiardi \& Codazzi, 2009; Lingiardi \& Tanzilli, 2011).

\section{Relazione terapeutica e sue componenti: alleanza, transfert, controtransfert}

Il volume L'alleanza terapeutica (Lingiardi, 2002), rassegna storicocritica delle principali definizioni teoriche e operazionalizzazioni empiriche del costrutto, inaugura in Italia una prolifica linea di ricerca, concettuale ed empirica, su questo "fattore aspecifico", tra i più correlati all'outcome delle psicoterapie (Horvath \& Bedi, 2002; Lingiardi, 2006a; Wampold, 2001). La prima ricerca che abbiamo svolto in quest'ambito si è concentrata sullo studio della relazione tra 
disturbi di personalità e alleanza terapeutica (Lingiardi, Filippucci, \& Baiocco, 2005). In questo lavoro abbiamo potuto osservare come (a) la forza dell'alleanza terapeutica nelle fasi iniziali del trattamento fosse un buon predittore rispetto al drop out; (b) i pazienti del cluster A mostrino particolari difficoltà nello stabilire un'alleanza terapeutica; (c) i terapeuti con pazienti del cluster B tendano a valutare negativamente l'alleanza e, infine, (d) le valutazioni dell'alleanza fatte dai terapeuti risultino in media più basse di quelle fatte dai pazienti.

All'interno di una riflessione, anche critica, sulla "genericità" del costrutto "alleanza terapeutica", la nostra attenzione si è quindi spostata sullo studio della costruzione dell'alleanza nel corso del processo terapeutico (Ackermann \& Hilsenroth, 2001, 2003) e sulla concettualizzazione di Safran e Muran secondo cui l'alleanza può essere intensa come processo di negoziazione intersoggettiva tra paziente e terapeuta caratterizzato dall'alternarsi di rotture e riparazioni (Lingiardi, 2008, in press; Lingiardi \& Colli, 2010; Safran \& Muran, 2000). A partire da questo modello abbiamo costruito l'Indice di Valutazione dell'Alleanza Terapeutica (IVAT; Colli \& Lingiardi, 2002, 2007a, 2007b), che permette di valutare i processi di rottura e riparazione dell'alleanza a partire dall'analisi dei trascritti di sedute secondo la prospettiva di un osservatore esterno e attraverso una metodologia microanalitica. Lo studio di validazione dell'ultima versione di questo strumento, la Collaborative Interaction Scale (CIS), è stata pubblicato su Psychotherapy Research (Colli \& Lingiardi, 2009a).

Con l'IVAT/CIS abbiamo studiato il rapporto tra processi di rottura e riparazione dell'alleanza terapeutica e variabili come: l'adjustment ratio degli interventi del terapeuta rispetto al funzionamento difensivo del paziente (Colli \& Lingiardi, 2006), il controtransfert (Lingiardi \& Colli, 2008), gli interventi del terapeuta (Lingiardi, Tanzilli, \& Colli, 2008), la diagnosi di personalità e i meccanismi di difesa (Lingiardi, Colli, \& Gazzillo, 2007), l'attività referenziale (Colli, Mariani, D’Angelo, \& 
Lingiardi, 2009; Condino, Colli, Mariani, \& De Coro, 2009) e i Sistemi Motivazionali Interpersonali valutati attraverso il metodo AIMIT (Liotti \& Monticelli, 2008; Colli, Gentile, Fassone, Ivaldi, Lingiardi, \& Liotti, 2010; Gentile, Colli, Fassone, Ivaldi, \& Liotti, 2009). Attualmente siamo impegnati nello sviluppo di una nuova versione user-friendly della CIS e nello studio della relazione tra rotture/riparazioni dell'alleanza e funzionamento riflessivo (Colli, Guardati, \& Pratesi, 2010).

Nel tentativo di integrare lo studio dei fattori relazionali aspecifici delle psicoterapie con quello dei fattori specifici, abbiamo indagato il rapporto tra alleanza, pattern d'interazione terapeutica e profondità dell'elaborazione (Lingiardi, Colli, Gentile, \& Tanzilli, submitted). In questa ricerca, condotta con lo Psychotherapy Process Q-set (PQS; Jones, 1985, 2000; Colli \& Gazzillo, 2006; Lingiardi \& Dazzi, 2008), abbiamo potuto rilevare due pattern d'interazione specifici legati all'outcome delle sedute: un pattern che descrive un'alleanza terapeutica intesa come "base sicura" (vedi anche paragrafo successivo), e un pattern che descrive un coinvolgimento attivo della coppia paziente-terapeuta nella regolazione della relazione nel qui e ora.

Abbiamo quindi avviato una serie di ricerche con l'obiettivo di ampliare lo studio del processo terapeutico ad altre variabili relazionali, come le comunicazioni di paziente e terapeuta, il transfert e il controtransfert, la personalità e lo stile difensivo. In particolare, una ricerca ha previsto l'applicazione delle Analytic Process Scales (APS; Waldron, Scharf, Hurst et al., 2004), insieme con la SWAP-200 e la DMRS, a 42 sedute di tre psicoterapie psicoanalitiche. I risultati di questa ricerca, presentati al convegno internazionale della Society for Psychotherapy Research svoltosi a Madison (Wisconsin) nel 2007 (Gazzillo \& Lingiardi, 2007), confermano i risultati di una precedente ricerca condotta negli Stati Uniti con le APS (Waldron, Scharf, Hurst et al., 2004, Waldron, Scharf, Crouse et al., 2004): la produttività delle 
comunicazioni del paziente correla con le sue capacità di buon funzionamento psicologico valutate con la SWAP-200 e con il livello di maturità delle difese valutato con la DMRS, ed è rafforzata dalle attività analitiche nucleari del terapeuta e della qualità dei suoi interventi.

Per poterci affidare a una valutazione empirica del controtransfert e del transfert, abbiamo costruito un sistema $Q$ sort per la valutazione dell'esperienza relazionale del terapeuta Countertransference Assessment $Q$ sort (CTA-Q sort; Colli \& Prestano, 2006; Colli \& Lingiardi, 2009b) e validato la versione italiana di due strumenti clinician-report: il Psychotherapy Relationship Questionnaire (PRQ; Bradley, Heim, \& Westen, 2005; Tanzilli, Colli, De Bei, \& Lingiardi, 2010) e il Countertransference Questionnaire (CTQ; Betan, Heim, Zittel Conklin, \& Westen, 2005; Tanzilli, Colli, \& Lingiardi, 2009).

La validazione del CTA-Q sort è stata effettuata all'interno di una ricerca nazionale, condotta con un gruppo di colleghi impegnati nei servizi pubblici e in studi privati, sullo studio dei pattern di esperienza transferale e controtransferale in relazione al livello di funzionamento e allo stile di personalità dei pazienti (Colli \& Lingiardi, 2009b; Colli, Lingiardi, \& Gruppo ASP, submitted). Questo studio ci ha permesso di costruire alcuni prototipi dell'esperienza controtransferale nel trattamento di pazienti con disturbi di personalità e abbiamo potuto osservare, in accordo con la letteratura internazionale (Rossberg, Karterud, Pedersen, \& Friis, 2007; Schwartz, Smith, \& Chopko, 2007), una stretta relazione tra livello sintomatologico e intensità dell'esperienza controtransferale dei terapeuti nonché la presenza di pattern di risposta dei terapeuti in relazione a specifici disturbi di personalità (Colli \& Lingiardi, 2009b; Colli, Marchegiani, \& Lingiardi, 2010; Lingiardi, 2008, in press).

Abbiamo valutato stabilità fattoriale, affidabilità e validità di costrutto delle versioni italiane del CTQ (trad. it. De Bei \& Lingiardi, 2006a; Tanzilli et al., 2009) e del PRQ (trad. it. De Bei, Lingiardi, 
2006b; Tanzilli et al., 2010). L'analisi fattoriale preliminare da noi eseguita su 160 CTQ mostra una struttura differente da quella rilevata dalle ricerche americane (Betan et al., 2005); abbiamo infatti individuato 5 fattori di controtransfert: positivo, impotente/frustrato, speciale/ipercoinvolto, sopraffatto/disorganizzato, distaccato/non coinvolto. Per testare l'applicabilità clinica della versione italiana di questo strumento è stata esaminata la relazione tra i 5 fattori del controtransfert e i fattori PD e Q della SWAP-200. In linea con le ipotesi di partenza, i disturbi del cluster A sembrano associati a un controtransfert distaccato; i disturbi del cluster B ai controtransfert impotente/frustrato e sopraffatto/disorganizzato e i disturbi del cluster C al controtransfert speciale/ipercoinvolto.

Per valutare la validità convergente sono state esaminate le correlazioni tra i 5 pattern controtransferali e le valutazioni dei trascritti di sedute di 14 coppie paziente-terapeuta condotte con lo Psychotherapy Process Q-set (PQS; Jones, 1985, 2000) e il Working Alliance InventoryObserver Form (WAI-O; Horvath, 1981, 1982; Horvath \& Greenberg, 1989): i dati hanno messo in evidenza come i fattori del controtransfert ipercoinvolto, distaccato e disorganizzato sembrino associati a una scarsa qualità dell'alleanza terapeutica. Rispetto alle variabili del processo terapeutico, invece, la presenza di un pattern controtransferale positivo si associa a un legame di fiducia e intimità col paziente (Tanzilli et al., 2009).

Per quanto riguarda il Psychotherapy Relationship Questionnaire, l'analisi fattoriale eseguita su 180 protocolli ha prodotto una soluzione a 4 fattori differente da quella identificata dal gruppo di Westen (Bradley et al., 2005): sicuro-impegnato, arrabbiato/ostile, evitante, ansioso/preoccupato. Queste dimensioni transferali presentano elementi di sovrapposizione con gli stili di attaccamento identificabili attraverso la Adult Attachment Interview (AAI; Main, Kaplan, \& Cassidy, 1985). Anche in questo studio, per testare la validità e l'applicabilità 
clinica della versione italiana del PRQ è stata indagata la relazione tra $\mathrm{i}$ 4 fattori transferali e i disturbi della personalità alla SWAP-200 (sia in fattori $\mathrm{PD}$, sia in fattori Q). In accordo con le ipotesi formulate, $\mathrm{i}$ disturbi del cluster A sono risultati associati positivamente al transfert evitante/controdipendente e negativamente al transfert sicuro/impeganto; i disturbi del cluster B positivamente al transfert arrabbiato/ostile e negativamente al transfert sicuro/impegnato; i disturbi del cluster $\mathrm{C}$ positivamente al transfert ansioso/preoccupato e negativamente al transfert evitante/controdipendente.

Per valutare la validità convergente del PRQ sono state misurate le correlazioni tra i pattern transferali e le valutazioni dei trascritti di sedute di 20 coppie paziente-terapeuta condotte con il PQS di Jones (1985, 2000) e il WAI-O di Horvath (Horvath, 1981, 1982; Horvath \& Greenberg, 1989). I risultati preliminari di questa ricerca evidenziano come $\mathrm{i}$ fattori transferali ostile ed evitante siano associati a una bassa qualità dell'alleanza terapeutica, mentre il transfert positivo si associa a caratteristiche qualitativamente positive dell'interazione terapeutica, come la sua capacità del paziente di impegnarsi nel lavoro terapeutico, di essere introspettivo e di esplorare i propri pensieri e sentimenti (Gentile \& Tanzilli, 2008; Lingiardi et al., submitted; Tanzilli et al., 2010).

\section{Attaccamento e psicoterapia}

Le numerose ricerche che si sono occupate di estendere alla relazione terapeutica i risultati degli studi sull'attaccamento adulto (Obegi \& Berant, 2008; Slade, 2008; Steele \& Steele, 2008) ci hanno portato ad approfondire, da un punto di vista teorico e empirico, il rapporto tra questo costrutto e l'alleanza terapeutica, il transfert e il controtransfert (De Bei, Colli, \& Lingiardi, 2007; Lingiardi, 2002; Lingiardi \& De Bei, 2005, 2007) senza per questo tralasciare una riflessione sul tema, più ampio, del setting terapeutico (vedi Lingiardi $\&$ 
De Bei, 2008; Lingiardi \& De Bei, in press).

In più occasioni abbiamo sottolineato l'utilità di un ampliamento del concetto di alleanza in termini di "base sicura" (De Bei et al., 2007; Lingiardi \& De Bei, 2005, 2007). Lo studio micro-processuale della relazione terapeutica ha infatti messo in evidenza come l'alleanza rappresenti un costrutto sovraordinato che deriva dall'integrazione di numerose variabili riconducibili alle dimensioni transferale/controtransferale e relative alla relazione reale. Se si concepisce il transfert come ricapitolazione delle modalità relazionali del paziente (sicure o insicure), e i processi controtransferali come il risultato dell'incontro tra le caratteristiche della personalità del terapeuta e le particolari modalità relazionali del paziente, l'alleanza può essere compresa come risultante dell'incontro tra questi fattori e la dimensione reale e operativa della relazione terapeutica. Ciò consente di mettere in evidenza come l'alleanza, una volta concepita come base sicura, possa rappresentare un ponte tra costrutti apparentemente lontani.

Sul piano valutativo, al fine indagare il modo in cui l'attaccamento influenza l'outcome della psicoterapia (De Bei, 2006) abbiamo costruito uno strumento che permette di indagare l'attaccamento pazienteterapeuta a partire da trascritti delle sedute. Il Patient-Therapist Attachment Q- sort (PTA Q- sort; De Bei, Lingiardi, \& Miccoli, 2007) è uno strumento costruito con il preciso intento di studiare gli aspetti processuali delle dinamiche di attaccamento. I primi studi di validazione che abbiamo condotto hanno dato risultati incoraggianti dimostrando che lo strumento possiede un'affidabilità soddisfacente (De Bei, Lingiardi, \& Miccoli, 2008). Attualmente, il nostro gruppo di ricerca è impegnato a valutare la validità di costrutto del PTA Q- sort con altre misure dell'attaccamento adulto (AAI) e a costruirne una versione informatizzata. 
Sul piano empirico, l'impiego del PTA Q- sort ci ha consentito di verificare alcune ipotesi presenti in letteratura sulla relazione tra attaccamento paziente-terapeuta e outcome del trattamento (Meyer \& Pilkonis, 2001; Speranza, 2006). A questo scopo abbiamo indagato il rapporto tra la qualità dell'attaccamento paziente-terapeuta (De Bei, Lingiardi, \& Miccoli, 2007), l'alleanza (con il Working Alliance Inventory, WAI; Horvath, 1981, 1982; Horvath \& Greenberg, 1989) e le caratteristiche dell'interazione tra clinico e paziente (attraverso lo Psychotherapy Process Q-set, PQS; Jones, 1985, 2000). Questo studio, condotto su un campione di circa 40 sedute di psicoterapia a orientamento psicodinamico, sembra mostrare che l'attaccamento non è una variabile in grado di influenzare direttamente l'alleanza, ma alcuni fattori che, a loro volta, incidono sull'alleanza (De Bei, Tanzilli, Miccoli, \& Lingiardi, 2009). In sintesi, le modalità di interazione associate ai singoli pattern di attaccamento paziente-terapeuta sembrano catturare stili di interazione globali capaci di influenzare gli aspetti più tecnici dell'intervento mediando fattori aspecifici e specifici (Norcross, 2002; vedi anche Castonguay \& Butler, 2006).

Abbiamo applicato il PTA Q sort allo studio di un caso singolo, cosi da indagare un'ipotesi da poco presente in letteratura: alcuni studi che hanno utilizzato l'AAI come misura di outcome (Diamond, Clarkin, Stovall-Mcclough, Levy, Foelsch, Levine, \& Yeomans, 2003) hanno rilevato che un sottogruppo di pazienti borderline, nel corso del primo anno di psicoterapia, passavano da stati della mente disorganizzati/insicuri a stati non classificabili; mostravano, cioè, quello che poteva essere intepretato come un peggioramento. Si è tuttavia ipotizzato (Speranza, 2006) che questo dato rifletta una "rottura" delle precedenti strategie insicure che nel prosieguo della terapia può condurre allo sviluppo di modalità di relazione più sicure. Lo studio che abbiamo condotto su una psicoterapia psicodinamica della durata di due anni ha indicato di fatto un andamento a 
"campana" delle misure di attaccamento, con un aumento delle caratteristiche di insicurezza nella fase intermedia della terapia a cui fa seguito, nella fase finale, un incremento della sicurezza. Questo dato, certo non generalizzabile, sembra muoversi in accordo con l'ipotesi per cui un'acquisizione di strategie più sicure nel corso di una psicoterapia possa passare attraverso una "rottura" delle precedenti strategie insicure (De Bei, Lingiardi, \& Miccoli, 2008).

La possibilità di monitorare in senso dimensionale l'andamento delle diverse strategie di attaccamento (preoccupate, distanzianti e sicure) ci ha consentito di evidenziare come esse possano essere lette in termini di modalità di regolazione degli affetti. Nello studio single case sopracitato abbiamo infatti rilevato una brusca diminuzione del fattore distanziante e un concomitante incremento del fattore preoccupato nella fase intermedia della terapia e un ritorno a livelli precedenti di distanziamento, ma inseriti nel contesto di una maggior sicurezza e di una minore preoccupazione, nella fase finale. Se si leggono distanziamento e preoccupazione come una iper-regolazione (il primo) e una ipo-regolazione (il secondo) delle emozioni, l'andamento evidenziato può essere descritto nei termini dell'acquisizione di una modalità più funzionale di regolazione degli affetti.

Questi risultati ci hanno spinto ad approfondire lo studio dell'attaccamento paziente-terapeuta estendendolo ad altre variabili processuali (per esempio, i meccanismi di difesa; De Bei \& Tanzilli, 2010) e ad avviare una riflessione concettuale e teorica sull'impiego clinico di questa variabile (Dazzi \& De Bei, 2010).

\section{La valutazione dei meccanismi di difesa}

Il nostro interesse per questo costrutto nasce con la scrittura di un volume di rassegna sistematica dei principali modelli teorici e strumenti empirici per la valutazione dei meccanismi di difesa 
(Lingiardi, 2006b; Lingiardi \& Madeddu, 2002), contenente tra l'altro la versione italiana della Defense Mechanism Rating Scale (DMRS; Perry, 1991), da noi validata su un campione di pazienti con disturbi di personalità (Lingiardi, Lonati, Fossati, Vanzulli, \& Maffei, 1999). La DMRS è stata impiegata in molte ricerche italiane su processo e outcome delle psicoterapie di pazienti adulti (vedi paragrafi 1 e 2).

Un altro strumento per la valutazione dei meccanismi di difesa da noi tradotto e validato è il Defense Style Questionnaire (DSQ; San Martini, Roma, Sarti, Lingiardi, \& Bond, 2004), strumento self-report per la valutazione dei meccanismi di difesa costruito negli anni Ottanta da Michael Bond (Bond, 1986).

Al fine di estendere i benefici della valutazione dei meccanismi di difesa anche al lavoro clinico, dove spesso risulta difficoltoso applicare le complesse procedure di rating proposte dagli strumenti più utilizzati (Cramer, 2007; Perry, 1991) abbiamo realizzato, in collaborazione con il gruppo di ricerca di Perry, un nuovo sistema di valutazione ispirato alla DMRS, ma di più facile somministrazione e codifica, adatto sia per scopi clinici che di ricerca (Perry, Di Giuseppe, Petraglia, Janzen, \& Lingiardi, submitted): il DMRS Q-sort version (DMRS-Q). Il DMRS-Q è composto da 150 item relativi a 30 meccanismi di difesa organizzati gerarchicamente in 7 livelli di adattività/maturità. Al valutatore si chiede di organizzare le 150 affermazioni all'interno di 7 ranghi di descrittività secondo una distribuzione fissa, in modo da ottenere un quadro preciso di quali manifestazioni difensive possano essere considerate caratterizzanti un determinato individuo. Disponendo di 5 item per ogni singolo meccanismo di difesa, ognuno dei quali sottolinea un particolare aspetto funzionale e modalità espressiva della difesa sottostante, il Q-sort è in grado di catturare le caratteristiche dello stile difensivo sia qualitativamente, tracciando un Defensive Profile, sia quantitativamente, attraverso i punteggi per singola difesa, livello difensivo e punteggio di maturità difensiva globale (Overall Defensive 
Functioning - ODF). L'intera procedura di codifica e interpretazione dei risultati è facilitata da un software appositamente ideato (Di Giuseppe, 2009).

Tenendo conto del fatto che l'adolescenza può essere considerata l'età in cui si stabilizzano i pattern cognitivi, affettivi e comportamentali che andranno a formare la personalità adulta, abbiamo deciso di affrontare in modo empirico anche lo studio dei meccanismi di difesa in soggetti adolescenti. Molti studi sulla relazione tra stile difensivo e caratteristiche di personalità hanno mostrato come il ricorso a difese mature si associ a benessere fisico e psicologico, mentre meccanismi di difesa meno adattivi e immaturi siano tipici dei soggetti con disturbi di personalità (Perry, Lingiardi, \& Ianni, 1999; Bond \& Perry, 2004; Vaillant, 1992). Sulla scorta dei risultati emersi dagli studi con la SWAP-200 (vedi anche paragrafo 1), abbiamo rilevato l'opportunità di indagare la relazione tra meccanismi di difesa e caratteristiche di personalità in adolescenza, evidenziandone gli aspetti di continuità e discontinuità con i risultati ottenuti in campioni di adulti (Vaillant \& Drake, 1985; Lingiardi, Lonati, Fossati, Vanzulli, \& Maffei, 1999; Cramer, 1999) e verificando la validità e attendibilità di metodi empirici per la loro valutazione (come la DMRS e il DMRS-Q) anche su popolazioni in età evolutiva (Di Giuseppe, Ianni, Gazzillo, \& Lingiardi, 2009).

Abbiamo quindi condotto una ricerca su 42 soggetti di età compresa tra i 14 e i 18 anni in trattamento presso servizi pubblici per la prevenzione delle problematiche psicologiche e sociali dell'età evolutiva. La valutazione della personalità è stata effettuata con la SWAP-200-A a partire dai trascritti dall'Intervista Clinica e Diagnostica (CDI; Westen \& Muderrisoglu, 2003), di cui abbiamo elaborato una versione per adolescenti (Gazzillo, 2006), sui quali è stata applicata anche la DMRS e la sua versione Q-sort (Di Giuseppe, Perry, Petraglia, Janzen, \& Lingiardi, 2010). 
I primi risultati di questo studio mostrano che alcuni stili di personalità, in particolare quelli del Cluster B, si manifestano già in adolescenza con caratteristiche simili a quelle riscontrabili negli adulti. Inoltre, l'associazione con particolari meccanismi di difesa lascerebbe intuire che anche in adolescenza esiste una certa continuità tra i pattern difensivi e i disturbi di personalità, che solo più tardi si manifestano in forma stabile e strutturata (per una trattazione più specifica del problema della diagnosi di personalità in adolescenza, vedi Lingiardi, 2009; Lingiardi \& Codazzi, 2009, Lingiardi \& Maffei, 2009).

\section{Orientamento sessuale, omofobia, identità di genere e psicoterapia}

Partendo dallo studio dell'omofobia sociale e interiorizzata (Lingiardi, 1992, 2007a, 2007b; Lingiardi \& Drescher, 2003; Lingiardi, Falanga, \& D’Augelli, 2005) abbiamo messo a punto due strumenti per la sua misurazione. In particolare, il questionario autosomministrato Measure of Internalized Sexual Stigma for Lesbians and Gay men (MISS-LG; Lingiardi, Baiocco, \& Nardelli, submitted; Nardelli, Baiocco, Rollè, \& Brustia, 2010) ci ha permesso di verificare l'ipotesi per cui l'omofobia interiorizzata sarebbe scomponibile in tre dimensioni: individuale, sociale, sessuale/diadica. Comprendere e poter disporre di un valido strumento per misurare il costrutto dell'omofobia interiorizzata è a nostro parere di estrema importanza nella ricerca e nelle psicoterapie con pazienti gay e lesbiche, spesso soggetti a un particolare tipo di stress - di cui l'omofobia interiorizzata fa parte - il minority stress (Lingiardi, 2007b; Meyer, 1995, 2003).

Abbiamo quindi messo in relazione l'omofobia interiorizzata e il minority stress con altri costrutti d'interesse clinico, quali la dissociazione e l'attaccamento. Più in dettaglio, i nostri studi sembrano indicare che alti livelli di omofobia interiorizzata predicono un maggior ricorso a strategie difensive di tipo dissociativo nelle persone gay e lesbiche (Lingiardi, Nardelli, Baiocco, \& Rollè, 2010; Nardelli, Baiocco, 
Rollè, \& Brustia, 2009). Per quanto riguarda l'attaccamento, i risultati indicano che alti livelli di omofobia interiorizzata si presentano spesso in associazione agli stili insicuri distanziante e timoroso (Feeney, Noller, \& Hanrahan, 1994; Fossati, Feeney, Donati, Donini, Novella, Bagnato, Acquarini, \& Maffei, 2003), a loro volta buoni predittori di omofobia interiorizzata (Lingiardi, Caristo, \& De Bei, submitted). Sempre in quest'area di ricerca, è in corso uno studio volto a comprendere il ruolo di queste dimensioni nelle famiglie omoparentali. Data la centralità della dissociazione e dell'attaccamento nella pratica clinica, è nostro interesse approfondirne le implicazioni nel campo psicoterapeutico (Sherry, 2007).

Le nostre ricerche si sono poi focalizzate sugli effetti determinati dall'omofobia del terapeuta, e sugli effetti delle terapie volte a "convertire" l'omosessualità in eterosessualità (dette talvolta "terapie riparative"). Partendo da uno studio sugli atteggiamenti psicoanalitici verso le omosessualità, vincitore nel 2004 del Ralph Roughton American Psychoanalytic Association Paper Award (Lingiardi \& Capozzi, 2004), insieme agli Ordini degli Psicologi della Campania, del Lazio e del Piemonte abbiamo avviato una ricerca analoga rivolta agli psicologi. A tal fine, abbiamo costruito un questionario ad hoc, l'APO (Atteggiamenti degli Psicologi verso le Omosessualità; Lingiardi \& Nardelli, 2010) per studiare la relazione tra gli atteggiamenti degli psicologi verso le omosessualità e variabili quali il percorso formativo, il modello teorico di riferimento, l'esperienza clinica con pazienti omosessuali, etc.

Nel corso degli ultimi anni abbiamo avviato un filone di ricerca sulle caratteristiche di personalità dei pazienti con Disturbo dell'Identità di Genere (DIG). I nostri studi si sono focalizzati sul rapporto tra DIG, personalità e attaccamento (Lingiardi, De Bei, \& Covelli, 2009; De Bei, Covelli, Chianura, 2008) evidenzando la presenza di un numero di soggetti con DIG e attaccamento sicuro equiparabile a quello dei campioni non clinici, ma con una netta prevalenza di 
esperienze di abuso (fisico e/o sessuale) e perdite traumatiche nell'infanzia. Sul piano della personalità, i nostri dati hanno messo in evidenza la presenza di una generale difficoltà a livello di funzionamento sociale, interpersonale e affettivo tuttavia non inseribile in un quadro psicopatologico definito. Un dato che, con molta probabilità, riflette l'influenza delle esperienze di stigma e discriminazione sociale sul funzionamento psichico e relazionale di questi soggetti (Zucker, 2005).

\section{Conclusioni}

Crediamo che le ricerche elencate e in parte descritte in questo contributo riflettano la nostra visione della psicoterapia come relazione tra soggetti i cui esiti dipendono da fattori molteplici e complessi, specifici e aspecifici, che possono facilitare o ostacolare la crescita psicologica e il processo terapeutico. Le nostre riflessioni e applicazioni sui temi teorici e metodologici della ricerca in psicoterapia ha dato vita al primo manuale italiano in questo campo: La ricerca in psicoterapia. Modelli e strumenti (Dazzi, Lingiardi, \& Colli, 2006), patrocinato da SPR Italia e SPR International, introdotto dal Past President John Clarkin, e scritto in colloborazione con alcuni tra i principali gruppi di ricerca italiani.

\section{Bibliografia}

Ackerman, S.J., \& Hilsenroth, M. (2001). A review of therapist characteristics and techniques negatively impacting the therapeutic alliance. Psychotherapy, 38(2), 171-183.

Ackerman, S.J., \& Hilsenroth, M. (2003). A review of therapist characteristics and techniques positively impacting the therapeutic alliance. Clinical Psychology Review, 23, 1-33.

American Psychiatric Association (2000). Manuale diagnostico e statistico dei disturbi mentali - Text revision (DSM-IV-TR). Tr. it. Masson, Milano 2002. 
Ricerca in Psicoterapia/Research in Psychotherapy 2010; 2(13): 97-124 http://www.researchinpsychotherapy.net

Barron, J., \& Lingiardi, V. (2005). Prefazione all'edizione italiana. In J. Barron (Ed.), Dare un senso alla diagnosi (pp. I-VIII). Tr. it. Raffaello Cortina, Milano 2005.

Betan, E., Heim, A., Zittel Conklin, C., \& Westen, D. (2005). Countertransference phenomena and personality pathology in clinical practice: an empirical investigation. American Journal of Psychiatry, 162(5), 890-898.

Bond, M. (1986). Defense Style Questionnaire. In G.E. Vaillant (Eds.), Empirical studies of ego mechanisms of defense (pp. 146-152). Washington, DC: American Psychiatric Press.

Bond, M., \& Perry, J.C. (2004). Long-term changes in defense styles with psychodinamic psychotherapy for depressive, anxiety, and personality disorders. The American Journal of Psychiatry, 161(9), 1665-1671.

Bradley, R., Heim, A., \& Westen, D. (2005). Transference phenomena in the psychotherapy of personality disorders: an empirical investigation. British Journal of Psychiatry, 186, 342-349.

Bucci, W., \& Kabasakalian-Mckay, R. (1992). Scoring referential activity. Instructions for use with transcripts of spoken narrative texts (tr. it. in A. De Coro \& G. Caviglia (Eds.), La valutazione dell'attività referenziale. Kappa, Roma 2000).

Castonguay, L.G., \& Butler, L.E. (2006). Principles of Therapeutic Change: A task force on partecipants, relationship and techniques factors. Journal of Clinical Psychology, 62(5), 1-7.

Colli, A., \& Gazzillo, F. (2006). Due strumenti per la valutazione del processo terapeutico: le Analytic Process Scales e lo Psychotherapy Process Q-set. In N. Dazzi, V. Lingiardi \& A. Colli (Eds.), La ricerca in psicoterapia. Modelli e strumenti (pp. 677-699). Milano: Raffaello Cortina.

Colli, A., Gentile, D., Fassone, G., Ivaldi, A., Lingiardi, V., \& Liotti, G. (2010, Ottobre). Rotture e riparazioni dell'alleanza terapeutica e sistemi motivazionali interpersonali: uno studio esplorativo con i metodi CIS e AIMIT. Relazione presentata al VIII Congresso Nazionale SPR-Italia, Perugia.

Colli, A., Guardati, C., \& Pratesi, D. (2010, Settembre). Rotture e riparazioni dell'alleanza terapeutica, difese e funzione riflessiva: uno studio pilota su tre casi singoli. Relazione presentata al Convegno Nazionale AIP, Sezione Dinamica e Clinica, Torino.

Colli, A., \& Lingiardi, V. (2002). Indice di valutazione dell'alleanza terapeutica (IVAT). Una proposta di valutazione dell'alleanza terapeutica a partire dai trascritti delle sedute. In V. Lingiardi, L'alleanza terapeutica. Teoria, clinica, ricerca (pp. 211-222). Milano: Raffaello Cortina.

Colli, A., \& Lingiardi, V. (2006, June). Therapist's interventions adjustment ratio and relational failures: A pilot study on the way therapist breaks the alliance. Paper presented at the $37^{\text {th }}$ International Meeting of the Society for Psychotherapy Research, Edinburgh, Scotland.

Colli, A., \& Lingiardi, V. (2007a). Valutare l'alleanza terapeutica attraverso trascritti di sedute: l'attendibilità della IVAT-R nello studio di quattro psicoterapie. Ricerca in psicoterapia, 10(1), 75-97.

Colli, A., \& Lingiardi, V. (2007b, June). Reliability and validity of a new measure to assess therapeutic alliance ruptures and resolutions: the Therapeutic Alliance Index- $R(T A I-R)$. Paper presented at the $38^{\text {th }}$ Annual Meeting of the Society for Psychotherapy Research, Madison, USA. 
Ricerca in Psicoterapia/Research in Psychotherapy 2010; 2(13): 97-124 http://www.researchinpsychotherapy.net

Colli, A., \& Lingiardi, V. (2009a). The Collaborative Interactions Scale: A new transcript-based method for the assessment of therapeutic alliance ruptures and resolutions in psychotherapy. Psychotherapy Research, 19(6), 718-734.

Colli, A., \& Lingiardi, V. (2009b, October). Toward the construction of empirically derived countertransference prototypes: The countertrasference assessment $Q$ - sort. Paper presented at the $7^{\text {th }}$ European Meeting SPR, Bozen.

Colli, A., Lingiardi, V., \& Gruppo clinico di ricerca ASP (submitted). Toward the construction of empirically derived countertransference prototypes: a study based on a Q sort methodology.

Colli, A., Marchegiani, M., \& Lingiardi, V. (2010, Ottobre). Profili discriminativi controtransferali nel trattamento di pazienti con disturbi di personalità. Relazione presentata al IX Congresso Nazionale SPR-Italia, Perugia.

Colli, A., Mariani, R., D’Angelo, V., \& Lingiardi, V. (2009, Aprile). Studio esplorativo sui processi di rottura dell'alleanza in relazione alle comunicazioni emozionali nel processo referenziale. Relazione presentata alla giornata di studio "Il processo referenziale. Studi clinici e ricerca empirica", Sapienza Università di Roma, Roma. Atti del convegno pubblicati in Quaderni CDP, 6, 139-152.

Colli, A., \& Prestano, C. (2006). La ricerca empirica sul controtransfert. In N. Dazzi, V. Lingiardi \& A. Colli (Eds.), La ricerca in psicoterapia. Modelli e strumenti (pp. 301-328). Milano: Raffaello Cortina.

Condino, V., Colli, A., Mariani, R., \& De Coro, R. (2009, Settembre). Studio sui processi di rottura dell'alleanza terapeutica in relazione alle comunicazioni emozionali nel processo referenziale. Relazione presentata al Convegno Nazionale AIP - Sezione Clinica, Chieti.

Cramer P. (1999) Ego functions and ego development: defense mechanisms and intelligence as predictors of ego level. Journal of Personality, 67(5), 73560.

Cramer, P. (2007) Longitudinal study of defense mechanisms: late childhood to late adolescence. Journal of Personality, 75(1), 1-24.

Dazzi, N., \& De Bei, F. (2010). Introduzione all'edizione italiana. In J. Cassidy $\&$ P. Shaver, (Eds.), Manuale dell'attaccamento (seconda edizione) (tr. it. Giovanni Fioriti, Roma 2008).

Dazzi, N., Lingiardi, V., \& Colli, A. (Eds.). (2006). La ricerca in psicoterapia. Modelli e strumenti. Milano: Raffaello Cortina.

Dazzi, N., Lingiardi, V., \& Gazzillo, F. (Eds.). (2009). La diagnosi in psicologia clinica. Milano: Raffaello Cortina.

De Bei, F. (2006). La ricerca sull'alleanza terapeutica. In N. Dazzi, V. Lingiardi $\&$ A. Colli (Eds.). La ricerca in psicoterapia. Modelli e strumenti (pp. 505537). Milano: Raffaello Cortina.

De Bei F., Colli A., \& Lingiardi V. (2007). Decostruire la relazione terapeutica per ricostruirla. Psicologia clinica dello sviluppo, 1, 50-69.

De Bei, F., Covelli, G., \& Chianura, L. (2008, Settembre). Personalità e attaccamento in adulti con disturbo dell'identità di genere. Relazione presentata al XI Congresso Nazionale dell'Associazione Italiana di Psicologia (AIP), Padova.

De Bei, F., \& Lingiardi, V. (2006a). Traduzione italiana del Countertransference Questionnaire (CTQ). Dipartimento di Psicologia Dinamica e Clinica, "Sapienza”, Università degli Studi di Roma. 
Ricerca in Psicoterapia/Research in Psychotherapy 2010; 2(13): 97-124 http://www.researchinpsychotherapy.net

De Bei, F., \& Lingiardi, V. (2006b). Traduzione italiana del Psychotherapy Relationship Questionnaire (PRQ). Dipartimento di Psicologia Dinamica e Clinica, "Sapienza", Università degli Studi di Roma.

De Bei, F., Lingiardi, V., \& Miccoli, D. (2007). Patient Therapist Attachment Qsort (PTA-Q). Manuale non pubblicato. Dipartimento di Psicologia Dinamica e Clinica, "Sapienza", Università degli Studi di Roma.

De Bei, F. Lingiardi, V., \& Miccoli, D. (2008, Ottobre). Relazione terapeutica, attaccamento e processo terapeutico: il Patient Therapist Attachment Q-sort (PTA-Q). Relazione presentata al VII Convegno SPR-Italia, Modena.

De Bei, F., \& Tanzilli, A. (2010, Settembre). Attaccamento e meccanismi di difesa nella relazione terapeutica: una studio empirico. Relazione presentata al VI Congresso Nazionale della Sezione di Psicologia Dinamica e Clinica, Torino.

De Bei, F., Tanzilli, A., Miccoli, D., \& Lingiardi, V. (2009, October). Therapeutic relationship, attachment and therapeutic process: an empirical study. Paper presented at the VII European Conference SPR, Bozen.

Diamond, D., Clarkin, J.F., Stovall-Mcclough, C., Levy, K.N., Foelsch, P.A., Levine, H., \& Yeomans, F.E. (2003). Patient-Therapist attachment: impact onthe therapeutic process and outcome. In M. Cortina \& M. Marrone (Eds.), Attachment Theory and the Psychoanalytic Process (pp. 127-178). London: Whurr Publishers.

Di Giuseppe, M. (2009). Verso una versione Q-sort della DMRS: dati preliminari. In M. Vigorelli (Ed.), Laboratorio didattico per la ricerca in psicoterapia (pp. 161-163). Milano: Raffaello Cortina.

Di Giuseppe, M., Ianni, L., Gazzillo, F., \& Lingiardi, V. (2009, October). Defense mechanisms and personality psychopatology in adolescence. A correlation study between DMRS and SWAP-200. Paper presented at 7 th European Meeting of Society for Psychotherapy Research (SPR), Bozen.

Di Giuseppe, M., Perry, J.C., Petraglia, J., Janzen, J., \& Lingiardi, V. (2010, June). Studying change in defensive functioning using the new computerized defense mechanism rating scale $Q$-sort (DMRS Q-sort) in psychotherapy. Paper presented at $41^{\text {th }}$ International Meeting of Society for Psychotherapy Research, Asilomar, CA.

Feeney, J.A., Noller, P., \& Hanrahan, M. (1994). Assessing adult attachment. In M.B. Sperling \& W.H. Berman (Eds.), Attachment in adults: Clinical and Developmental Perspectives (pp. 128-154). New York: Guilford Press.

Fossati, A., Feeney, J.A., Donati, D., Donini, M., Novella, L., Bagnato, M., Acquarini, E., \& Maffei, C. (2003). On the dimensionality of the Attachment Style Questionnaire in Italian clinical and non clinical partecipans. Journal of Social and Personal Relationships, 20, 55-79.

Gazzillo, F. (2006). La valutazione della personalità e la ricerca in psicoterapia: la SWAP-200 (pp. 609-628). In N. Dazzi, V. Lingiardi \& A. Colli (Eds.), La ricerca in psicoterapia. Modelli e strumenti. Milano: Raffaello Cortina.

Gazzillo, F. (2009). La Shedler-Westen-Assessment Procedure (SWAP). In N. Dazzi, V. Lingiardi \& F. Gazzillo (Eds.), La diagnosi in psicologica clinica: personalità e psicopatologia. Milano: Raffaello Cortina.

Gazzillo, F., \& Lingiardi, V. (2007, June). The virtuous circle of a good analysis. Paper presented at the $38^{\text {th }}$ international meeting of the Society for Psychotherapy Research, Madison, Wisconsin. 
Ricerca in Psicoterapia/Research in Psychotherapy 2010; 2(13): 97-124 http://www.researchinpsychotherapy.net

Gazzillo, F., \& Lingiardi, V. (2010). Prototipi Diagnostici Psicodinamici. Versione definitiva con scoring computerizzato. Dipartimento di Psicologia Dinamica e Clinica, "Sapienza", Università degli Studi di Roma.

Gazzillo, F., Lingiardi, V., \& Cordero di Montezemolo, C. (2008). Prototipi diagnostici psicodinamici (PDP). Manoscritto non pubblicato. Dipartimento di Psicologia Dinamica e Clinica, "Sapienza", Università degli Studi di Roma.

Gazzillo, F., Lingiardi, V., \& Del Corno, F. (in progress). Toward the validation of a PDM Axis $\mathrm{P}$ based assessment instrument for personality disorders.

Gazzillo, F., Lingiardi, V., Peloso, A., Giordani, S., Vesco, S., Filippucci, L., \& Zanna, V. (submitted). Personality subtypes of adolescents with personality disorders.

Gazzillo, F., Mariani, R., Lingiardi, V., \& Bucci, W. (2009). Verso un'integrazione tra personalità e attività referenziale: uno studio pilota su un caso singolo valutato con SWAP e RA. Relazione presentata alla giornata di studio "Il processo referenziale. Studi clinici e ricerca empirica", "Sapienza" Università di Roma, Roma. Atti del convegno pubblicati in Quaderni CDP, 6, 119-138.

Gentile, D., Colli, A., Fassone, G., Ivaldi, A., \& Liotti, G. (2009, Settembre). Rotture e riparazioni dell'alleanza terapeutica e sistemi motivazionali: uno studio esplorativo. Poster presentato al Convegno Nazionale AIP, Chieti.

Gentile, D., \& Tanzilli, A. (2008, Settembre). Profondità dell'elaborazione e alleanza terapeutica: un'indagine empirica. Premio Miglior Poster al VII Congresso Nazionale SPR-Italia, Modena.

Gruppo per lo studio delle motivazioni interpersonali in psicoterapia (2008). Il manuale AIMT. In G. Liotti \& F. Monticelli (Eds.), I sistemi motivazionali nel dialogo clinico. Il manuale AIMIT (pp. 157-214). Milano: Raffaello Cortina.

Horvath, A.O. (1981). An exploratory study of the working alliance: Its measurement and relationship to outcome. Unpublished manuscript. University of British Columbia, Vancouver, Canada.

Horvath, A.O. (1982). Working Alliance Inventory (Revised), Unpublished manuscript. Simon Fraser University.

Horvath, A.O., \& Bedi, R.P. (2002). The alliance. In J. Norcross (Ed.), Psychotherapy relations that work. New York: Oxford.

Horvath, A.O., \& Greenberg, L.S. (1989). Development and validation of the Working Alliance Inventory. Journal of Counseling Psychology, 36, 223-233.

Jones, E.E. (1985). Manual for the Psychotherapy Process Q-sort. Unpublished manuscript. Berkeley, CA: University of California.

Jones, E.E. (2000). Therapeutic Action. A guide of psychoanalytic therapy. Northvale, NJ: Jason Aronson (tr. it. L'azione terapeutica. Una guida alla terapia psicoanalitica, Raffaello Cortina, Milano 2008).

Liotti, G., \& Monticelli, F. (2008). I sistemi motivazionali nel dialogo clinico. Il manuale AIMIT. Milano: Raffaello Cortina.

Lingiardi, V. (1997). Compagni d'amore. Milano: Raffaello Cortina,

Lingiardi, V. (2002). Men in Love. Chicago: Open Court.

Lingiardi, V. (2002). L'alleanza terapeutica. Teoria, clinica, ricerca. Milano: Raffaello Cortina.

Lingiardi, V. (2004). La personalità e i suoi disturbi. Lezioni di psicopatologia dinamica. Milano: Il Saggiatore.

Lingiardi, V. (2006a). Alleanza terapeutica. In F. Barale, M. Bertani, V. Gallese, S. Mistur \& A. Zamperini (Eds.), Dizionario storico di psicologia, psichiatria, psicoanalisi, neuroscienze (pp. 36-38). Torino: Einaudi. 
Ricerca in Psicoterapia/Research in Psychotherapy 2010; 2(13): 97-124 http://www.researchinpsychotherapy.net

Lingiardi, V. (2006b). Meccanismi di difesa. In F. Barale, M. Bertani, V. Gallese, S. Mistur \& A. Zamperini (Eds.), Dizionario storico di psicologia, psichiatria, psicoanalisi, neuroscienze (pp. 323-329). Torino: Einaudi.

Lingiardi, V. (2007a). Citizen gay. Famiglie, diritti negati, salute mentale. Milano: Il Saggiatore.

Lingiardi, V. (2007b). Dreaming gender. Restoration and Transformation. Studies in Gender and Sexuality, 8(4), 313-331.

Lingiardi, V. (2008). Playing with unreality: Transference and Computer. International Journal of Psychoanalysis, 89(1), 111-126.

Lingiardi, V. (2009). Quale diagnosi di personalità in adolescenza? Psichiatria dell'infanzia e dell'adolescenza. Nuova serie, 76(1), 127-145.

Lingiardi, V. (2011, in press). Realities in dialogue. Psychoanalytic Dialogues.

Lingiardi, V., Baiocco, R., \& Nardelli, N. (submitted). Measure of Internalized Sexual Stigma for Lesbians and Gay men: A new scale.

Lingiardi, V., \& Capozzi, P. (2004). Psychoanalytic attitudes towards homosexuality: An empirical research. International Journal of Psychoanalysis, 85, 137-158.

Lingiardi, V., Caristo, C., \& De Bei, F. (submitted). Attachment and Homophobia in gay and lesbian.

Lingiardi, V., \& Codazzi, A. (2009). La diagnosi di personalità in adolescenza: riflessioni introduttive e indicazioni metodologiche. Infanzia e Adolescenza, 8(3), 151-164.

Lingiardi, V., \& Colli, A. (2008, June). Therapist's countertransference and alliance rupture and resolution processes: an empirical investigation through the lens of Countertransference Assessment $Q$ sort (CTA $Q$ sort) and Collaborative Interactions Scale (CIS). Paper presented at the 39th Annual Meeting of the Society for Psychotherapy Research, Barcelona.

Lingiardi, V., \& Colli, A. (2010). L'alleanza terapeutica nelle psicoterapie psicodinamiche. Psicobiettivo, 1, 28-53.

Lingiardi, V., Colli, A., \& Gazzillo, F. (2007). Diagnosi di personalità, alleanza terapeutica e meccanismi di difesa. In G. Nicolò \& S. Salvatore (Eds.), La ricerca sui risultati e sul processo in psicoterapia (pp. 229-250). Roma: Edizioni Carlo Amore.

Lingiardi, V., Colli, A., Gentile, D., \& Tanzilli, A. (submitted). Psychotherapy process and depth of elaboration.

Lingiardi, V., \& Dazzi, N. (2008). Enrico Jones: l'eredità clinica di un metodo empirico. In E.E. Jones (2000), L'azione terapeutica (pp. VII-XVIII). Tr. it. Raffaello Cortina, Milano 2008.

Lingiardi, V., \& De Bei, F. (2005). Rotture nell'alleanza: una lettura sistemicodiadica. Infanzia e Adolescenza, 4(2), 81-100.

Lingiardi, V., \& De Bei, F. (2007). Alleanza terapeutica, transfert, controtransfert, attaccamento: come si integrano questi costrutti nella ricerca empirica sulla relazione terapeutica?. In G. Nicolò \& S. Salvatore (Eds.). La ricerca in psicoterapia. Roma: Carlo Amore Edizioni.

Lingiardi, V., \& De Bei, F. (2008). L'uso del lettino nella psicoanalisi contemporanea: revisione della letteratura e una ricerca empirica. Psicoterapia e Scienze Umane, XLII(3), 313-350.

Lingiardi, V., \& De Bei, F. (in press). Questioning the couch: theoretical and clinical perspective. Psychoanalytic Psychology. 
Ricerca in Psicoterapia/Research in Psychotherapy 2010; 2(13): 97-124 http://www.researchinpsychotherapy.net

Lingiardi, V., De Bei, F., \& Covelli, G. (2009, Novembre). Il disturbo dell'identità di genere: attaccamento, personalità e psicodinamica. Relazione presentata al VI Convegno Nazionale SAIFIP, Roma.

Lingiardi, V., \& Drescher, J. (Eds.). (2003). The Mental Health Professions and Homosexuality:International Perspectives. New York: The Haworth Medical Press.

Lingiardi, V., Falanga, S., \& D'Augelli, A. (2005), The evaluation of homophobia in an italian sample: An exploratory study. Archives of Sexual Behavior, 34(1), 81-94.

Lingiardi, V., Filippucci, L., \& Baiocco, R. (2005). Therapeutic alliance evaluation in personality disorders psychotherapy. Psychotherapy Research, 15(12), 45-53.

Lingiardi, V., \& Gazzillo, F. (2002). La valutazione della personalità secondo Drew Westen. Parte 1: La Shedler-Westen Assessment Procedure (SWAP-200 e SWAP-II). Infanzia e adolescenza, 1(2), 65-80.

Lingiardi, V., \& Gazzillo, F. (2010). Drew Westen e la ricerca in psicoterapia: un sostegno empirico al lavoro psicoanalitico. In A. De Coro \& F. Ortu (Eds.), Psicologia Dinamica. I modelli teorici a confronto (pp. 346-359). Bari-Roma: Laterza.

Lingiardi, V., Gazzillo, F., \& Waldron, S. (2010). An Empirically Supported Psychoanalysis: the Case of Giovanna. Psychoanalytic Psychology, 27(2), 190218.

Lingiardi, V., Lonati, C., Fossati, A., Vanzulli, L., \& Maffei, C. (1999). Defense Mechanisms and Personality Disorders. Journal of Nervous and Mental Disease, 187(4), 224-228.

Lingiardi, V., \& Madeddu, F. (2002). I meccanismi di difesa (nuova ed.). Milano: Raffaello Cortina.

Lingiardi, V., Madeddu, F., Fossati, A., \& Maffei, C. (1994). Reliability and Validity of the Personality Functioning Scale (PFS). Journal of Personality Disorders, 8(2), 111-120.

Lingiardi, V., \& Maffei, C. (2009). Adolescenza e disturbi di personalità. Infanzia e Adolescenza, 8(2), 67-72.

Lingiardi, V., \& Nardelli, N. (2010). L'atteggiamento degli psicologi del Lazio nei confronti dell'omosessualità: una ricerca pilota. Notiziario dell'Ordine degli psicologi del Lazio, 3 (in press).

Lingiardi, V., Nardelli, N., Baiocco, R., \& Rollè, L. (2010). Traumatic experience, dissociation and internalized sexual stigma in lesbians and gays. Manuscript submitted for publication.

Lingiardi, V., Shedler, J., \& Gazzillo, F. (2006). Assessing Personality Change in Psychotherapy with the SWAP-200: A Case Study. Journal of Personality Assessment, 86(1), 23-32.

Lingiardi, V., \& Tanzilli, A. (in press). La diagnosi psicodinamica in un'ottica contemporanea. Ricerca Psicoanalitica.

Lingiardi, V., Tanzilli, A., \& Colli, A. (2008). Interventi del terapeuta e interazioni collaborative: alcune riflessioni sui processi di rottura e riparazione dell'alleanza terapeutica. Setting, 25, 12-35.

Maffei, C., Fossati, A., Lingiardi, V., Madeddu, F., Borellini, C., \& Petrachi, M. (1995). Personality maladjustment, defenses, and psychopathological symptoms in nonclinical subjects. Journal of Personality Disorders, 9(4), 330-345. 
Ricerca in Psicoterapia/Research in Psychotherapy 2010; 2(13): 97-124 http://www.researchinpsychotherapy.net

Main, M., Kaplan, N., \& Cassidy, J. (1985). Security in Infancy, Childhood and Adulthood: A Move to the Level of Representation. Monographs of the Society for Research in Child Development, no. 50. Chicago, IL: Chicago University Press.

Meyer, B., \& Pilkonis, P.A. (2001). Attachment style. Psychotherapy: Theory, Research, Practice, Training, 38(4), 466-472.

Meyer, I.H. (1995). Minority stress and mental health in gay men. Journal of Health and Social Behavior, 36(1), 38-56.

Meyer, I.H. (2003). Prejudice, social stress, and mental health in lesbian, gay, and bisexual populations: Conceptual issues and research evidence. Psychological Bulletin, 129, 674-697.

Nardelli, N., Baiocco, R., Rollè, L., \& Brustia, P. (2009, Settembre). Implicazioni dissociative dell'omonegatività interiorizzata. Relazione presentata al XI Convegno Nazionale dell'Associazione Italiana di Psicologia (AIP), Sezione di Psicologia Dinamica e Clinica, Chieti.

Nardelli, N., Baiocco, R., Rollè, L., \& Brustia, P. (2010, Settembre). La valutazione dello stigma sessuale interiorizzato nelle persone gay e lesbiche: La MISS-LG. Relazione presentata al XII Convegno Nazionale dell'Associazione Italiana di Psicologia (AIP), Sezione di Psicologia Dinamica e Clinica, Torino.

Norcross, J.N. (2002). Psychotherapy Relationships that Work. USA: Oxford University Press.

Obegi, J.H., \& Berant, E. (2008). Attachment Theory and Research in Clinical Work with Adults. London: Guilford Press.

PDM Task Force (2006). Manuale Diagnostico Psicodinamico (PDM). Tr. it. Raffaello Cortina, Milano 2008.

Perry, J.C. (1990). Defense Mechanism Rating Scale. Tr. it. in V. Lingiardi \& F. Madeddu (Eds.), I meccanismi di difesa (nuova ed.) (pp. 379-458). Raffaello Cortina, Milano 2002.

Perry, J.C. (1990). Defense Mechanism Rating Scales (DMRS) (fifth edition). Published by the author.

Perry, J.C. (1991). Defense Mechanisms Rating Scale. Cambridge Hospital, Harvard Medical School, Boston.

Perry, J.C., Di Giuseppe, M., Petraglia, J., Janzen, J., \& Lingiardi, V. (submitted). A preliminary study on validity and reliability of a Q-sort version of the Defense Mechanisms Rating Scales (DMRS-Q).

Perry, J.C., Lingiardi, V., \& Ianni, F. (1999). Psychodinamic research can help us to improve diagnosis and therapy for personality disorders: the case of defense mechanisms. In J. Derksen, H. Groen \& C. Maffei (Eds.), Treatment of Personality Disorders (pp. 39-52). New York: Kluwer Academic/Plenum Press.

Rossberg, J.I., Karterud, S., Pedersen, G., \& Friis, S. (2007). An empirical study of countertransference reactions toward patients with personality disorders. Comprensive Psychiatry, 48(3), 225-230.

Safran, J.D., \& Muran, C. (2000). Negotiating the therapeutic alliance: A relational treatment guide. New York: Guilford Press (tr. it. Teoria e pratica dell'alleanza terapeutica, Laterza, Bari 2003).

San Martini, P., Roma, P., Sarti, S., Lingiardi, V., \& Bond, M. (2004). Italian version of the Defense Style Questionnaire. Comprehensive Psychiatry, 45(6), 483-94. 
Ricerca in Psicoterapia/Research in Psychotherapy 2010; 2(13): 97-124 http://www.researchinpsychotherapy.net

Schwartz, R.C., Smith, S.D., \& Chopko, B. (2007). Psychotherapists' countertransference reactions toward clients with antisocila personality disorder and schizophrenia: an empirical investigation. American Journal of Psychotherapy, 61(4), 375-393.

Sherry, A. (2007). Internalized Homophobia and Adult Attachment: Implications for clinical practice. Psychotherapy: Theory, Research, Practice, Training, 44, 219-225.

Slade, A. (2008). The implications of attachment theory and research for adult psychotherapy: research and clinical perspectives. In J. Cassidy \& J. Shaver (Eds.), Handbook of attachment: Theory research and clinical applications (2th edition) (pp. 762-782). New York: Guilford Press.

Speranza, A.M. (2006). La valutazione dell'attaccamento. In N. Dazzi, V. Lingiardi \& A. Colli (Eds.), La ricerca in psicoterapia. Modelli e strumenti (pp. 539-566). Milano: Raffaello Cortina.

Steele, H., \& Steele, M. (2008). Clinical applications of the adult attachment interview. New York: Guilford Press (tr. it. Adult Attachment Interview. Applicazioni cliniche, Raffaello Cortina, Milano 2010).

Tanzilli, A., Carcione, A., Dimaggio, G., Lingiardi, V., \& Semerari, A. (2010, Ottobre). Transfert, controtransfert e patologia della personalità: un'indagine empirica. Relazione presentata al Convegno Nazionale SPR - Italia, Perugia.

Tanzilli, A., Colli, A., De Bei, F., \& Lingiardi, V. (2010, Settembre). Per una validazione italiana del Psychotherapy Relationship Questionnaire. Poster presentato al XVI Congresso Nazionale dell'Associazione Italiana di Psicologia (AIP), Sezione di Psicologia Clinica e Dinamica, Torino.

Tanzilli, A., Colli, A., \& Lingiardi, V. (2009, Settembre). Per una validazione italiana del Countertransference Questionnaire. Poster presentato al XV Congresso Nazionale dell'Associazione Italiana di Psicologia (AIP), Sezione di Psicologia Clinica e Dinamica. Chieti.

Thompson-Brenner, H., Eddy, K.T., Franko, D.L., Dorer, D.J., Vashchenko, M., Kass, A.E., \& Herzog, D.B.. (2008). A personality classification system for eating disorders: A longitudinal study. Comprehensive Psychiatry, 49, 551-560.

Thompson-Brenner, H., \& Westen, D. (2005). Personality subtypes in eating disorders: validation of a classification in a naturalistic sample. The British Journal of Psychiatry, 186, 516-524.

Vaillant, G.E. (1992). Ego mechanisms of defense: A guide for clinicians and researcher. Washington, DC: American Psychiatric Press.

Vaillant, G.E., \& Drake, R.E. (1985). Maturity of ego defenses in relation to DSM-III Axis II personality disorders. Archives of General Psychiatry, 42, 597-601.

Waldron, S., \& Helm, F. (2005). Psychodynamic features of two cognitivebehavioural and one psychodynamic treatment compared using the Analytic Process Scales. Canadian Journal of Psychoanalysis, 12, 346-368.

Waldron, S., Scharf, R.D., Crouse, J., Firestein, S.K., Burton, A., \& Hurst, D. (2004b). Saying the right thing at the right time: a view through the lens of the Analytic Process Scales (APS). Psychoanalytic Quarterly, 73, 1079-1125.

Waldron, S., Scharf, R.D, Hurst, D, Firestein, S.K., \& Burton, A. (2004a). What happens in a psychoanalysis: a view through the lens of the Analytic Process Scales (APS). International Journal of Psychoanalysis, 85, 443-466.

Wampold, B.E. (2001). The Great Psychotherapy Debate: Models, Methods, and Findings. Hillsdale, NJ: Erlbaum. 
Ricerca in Psicoterapia/Research in Psychotherapy 2010; 2(13): 97-124 http://www.researchinpsychotherapy.net

Westen, D. (2000). Psychotherapy Relationship Questionnaire (PRQ) manual. Vedi sito Internet http://www.psychsystems.net/lab.

Westen, D, \& Muderrisoglu, S. (2003). Reliability and validity of personality disorder assessment using a systematic clinical interview: Evaluating an alternative to structured interviews. Journal of Personality Disorders, 17, 350-368.

Westen, D., \& Shedler, J. (1999a). Revisione e valutazione dell'Asse II, Parte I: Lo sviluppo di un metodo clinicamente ed empiricamente valido. Tr. it. in D. Westen, J. Shedler \& V. Lingiardi (Eds.), La valutazione della personalità con la SWAP-200 (pp. 61-94). Milano: Raffaello Cortina.

Westen, D., \& Shedler, J. (1999b). Revisione e valutazione dell'Asse II, Parte II: Verso una classificazione dei disturbi della personalità empiricamente fondata e clinicamente utile. Tr. it. in D. Westen, J. Shedler \& V. Lingiardi (Eds.), La valutazione della personalità con la SWAP-200 (pp. 95-123). Milano: Raffaello Cortina.

Westen, D., Shedler, J., Durrett, C., Glass, S., \& Martens, A. (2003). Diagnosi di personalità in adolescenza: un'alternativa empiricamente derivata alle diagnosi dell'Asse II del DSM (tr. it. in Infanzia e Adolescenza, 3(1), 2004, 123).

Westen, D., Shedler, J., \& Lingiardi, V. (Eds.). (2003). La valutazione della personalità con la SWAP-200. Milano: Raffaello Cortina.

Zittel, C., \& Westen, D. (2003). The Countertransference Questionnaire. Emory University. Departments of Psychology and Psychiatry and Behavioral Sciences, Atlanta (vedi sito Internet http://www.psychsystems.net/lab).

Zucker, K.J. (2005). Gender Identity Disorder in children and adolescents. Annual Review of Clinical Psychology, 1, 467-492.

\section{Abstract}

The aim of this paper is to present an overview on the main research area of our group, coordinated by Vittorio Lingiardi. In particular we will focus on: a) personality diagnosis and assessment with SWAP-200 and PDM (Psychodynamic Diagnostic Manual); b) development and validation of PDM operazionalized clinician reports; c) assessment of defense mechanisms and defensive styles by DMRS and its Q sort version; d) the study of psychotherapy process and therapeutic relationship (i.e., therapeutic alliance, alliance ruptures and resolutions, transference, countertransference); in particular we developed a new measures for the assessment of ruptures and resolutions (the Collaborative Interaction Scale), and the quality of attachment between patient and therapist (the Patient-Therapist Attachment $Q$ Sort); d) the development of clinical and empirical research on gender identity, sexual orientation, social and internalized homophobia.

\section{Keywords}

Diagnosis and assessment of personality, defense mechanisms, process research, therapeutic alliance, sexual orientation and homophobia 